\title{
The Skin Remodeling in Type 1 Diabetes and Insulin Resistance Animal Models
}

\author{
M. KNAŚ ${ }^{1}$, K. WOLOSIK ${ }^{2}$, A. ZALEWSKA ${ }^{3}$, A. MIKUCKA-NICZYPORUK ${ }^{4}$, \\ I. KASACKA ${ }^{5}$, M. NICZYPORUK ${ }^{6}$
}

${ }^{1}$ Institute of Health Care, Higher Vocational School, Suwalki, Poland, ${ }^{2}$ Research Laboratory of Cosmetology, Faculty of Pharmacy with the Division of Laboratory Medicine, Medical University of Bialystok, Poland, ${ }^{3}$ Department of Pedodontics, Faculty of Medicine with Division of Dentistry, Medical University of Bialystok, Poland, ${ }^{4}$ Departments of Perinatology and Obstetrics, University Clinical Hospital of Bialystok, Poland, ${ }^{5}$ Departments of Histology and Cytophysiology, Faculty of Pharmacy with Division of Laboratory Medicine, Medical University of Bialystok, Poland, ${ }^{6}$ Research Laboratory of Esthetic Medicine, Faculty of Pharmacy with Division of Laboratory Medicine, Medical University of Bialystok, Poland

Received January 13, 2015

Accepted March 27, 2015

On-line June 5, 2015

\section{Summary}

The skin matrix metalloproteinase 3, tissue inhibitors of matrix metalloproteinase 2 and collagen III content changes in type 1 diabetes and insulin resistance treated with insulin and metformin were studied. Healthy adult male Wistar rats were obtained from experimental animal house, Department of Experimental Pharmacology, Medical University in Bialystok. The rats were divided randomly into five groups of 8 rats each. Control rats were injected intraperitoneally by $\mathrm{NaCl}$. Type IDDM was induced by a single injection of Streptozocin. Insulin resistance was induced by a high-fat diet. The chosen groups of rats were also treated with insulin or metformin. ELISA Kits (USCN Life Science, China) were used to measure content of matrix metalloproteinase 3 (ELISA Kit for Matrix Metalloproteinase 3 - MMP3), tissue inhibitor of matrix metalloproteinase 2 (ELISA Kit for Tissue Inhibitors of Metalloproteinase 2 - TIMP2) and content of collagen type 3 (ELISA Kit for Collagen Type III - COL3). The results were reported as a median. The statistical significance was defined as $p<0.05$. Type 1 diabetes and insulin resistance have significantly reduced the quality of the skin, shown by the increase in content of matrix metalloproteinase 3 and the decrease in content of tissue inhibitors of matrix metalloproteinase 2. Type 1 diabetes and insulin resistance have reduced the quality of the skin expressed by type III collagen content decrease but for future studies it is recommend to determine rat interstitial collagenase, MMP-13, as well. Insulin and metformin treatment improved the quality of the diabetic skin, demonstrated by the type III collagen content increase.

\section{Key words}

Type 1 diabetes • Insulin resistance • Insulin • Metformin

\section{Corresponding author}

K. Wołosik, Samodzielna Pracownia Kosmetologii, Uniwersytet Medyczny w Białymstoku, ul. Akademicka 3, 15-267 Białystok, Poland. E-mail: katarzyna.wolosik@umb.edu.pl

\section{Introduction}

Diabetes mellitus is a group of metabolic conditions characterized by hyperglycemia resulting from defects in insulin secretion, insulin action or both. The chronic hyperglycemia of people with diabetes is associated with long-term damage, dysfunction, and failure of different organs such as eyes, kidneys, nerves, heart and blood vessels (Powers 2005). One third of people with diabetes will have skin problems caused by diabetes mellitus. Such problems may be the first sign that a person has diabetes (Casqueiro et al. 2012). Many cutaneous signs are easily recognizable as diabetic 
markers and three: diabetic bullae, limited joint mobility and waxy skin, diabetic dermopathy are virtually diagnostic of diabetes (Jelinek 1994). Cutaneous manifestations of diabetes mellitus can be classified in the following categories: skin lesion due to diabetic abnormalities, e.g. diabetic microangiopathy, diabetic neuropathy, cutaneous infections, insulin resistance and various skin disorders (necrobiosis lipoidica, disseminated waxy skin granuloma annulare, diabetic bullae of diabetic mellitus, pruritis, stiff joints and waxy skin (Baloch 2008, Jelinek 1994), scleroderma (Lamba et al. 2005), vitiligo, lichen planus, hemochromatosis, eruptive xanthomas, finger pebbles, skin tags, local insulin reactions, insulin lipodystrophy, reactive perforating collagenosis-folliculitis (Baloch 2008, Jelinek 1994, Lamba et al. 2005). All patients with diabetes eventually develop skin complications from the long-term effects of diabetes mellitus on the microcirculation and on skin collagen; however patients with type 2 diabetes more often develop skin infections, whereas those with type 1 diabetes more often have autoimmune-related lesions (Powers 2005, Van Hattem et al. 2008). Type 1 diabetes mellitus (insulin-dependent diabetes mellitus IDDM) is a form that usually develops during childhood or adolescence and is characterized by severe deficiency of insulin secretion resulting from atrophy of the islets of Langerhans. The subsequent lack of insulin leads to increased blood and urine glucose. Type 2 diabetes mellitus (non-insulin-dependent diabetes mellitus NNIDM or adult-onset diabetes) (Powers 2005) is a metabolic condition that is characterized by hyperglycemia in the context of insulin resistance (IR) and relative lack of insulin (Masharani and German 2011). The relationships between insulin resistance and type 2 diabetes have been recognized. Insulin resistance is the most powerful predictor of future development of type 2 diabetes it is also a therapeutic target once hyperglycemia is present (Taylor 2012).

Early symptoms of insulin resistance may be missed, thus persons who are affected may not even know they have condition until type 2 diabetes occurs. Various skin defects are associated with insulin resistance including pseudo acanthosis nigricans (Lamba et al. 2005), hirsutism, acne, hidradenitis suppurativa, oiliness, alopecia, papulosis of the fingers and skin tags (de Almeida Tamega et al. 2010). Certain infections and sores that take a long time to heal are a warning sign of unrecognized diabetes. Typical biophysical properties of diabetic skin such as decreased skin elasticity, lower water content in stratum corneum, increased itching and sweating disturbances are reported (MackiewiczWysocka et al. 2014). The skin of diabetic patients has deficient wound healing properties caused by diabetic microangiopathy, neuropathy and disturbance in collagen metabolism. Some cutaneous conditions are linked in collagen changes (Jelinek 1994). Collagen is a major protein of the skin which plays an important role in the remodeling of skin structure. Skin tissue remodeling depends on the degradation of extracellular matrix (ECM) caused by the matrix metalloproteinases (MMPs) activity. MMPs activity is regulated by the tissue inhibitors of matrix metalloproteinases (TIMPs). The balance between MMPs and TIMPs is important to maintain homeostasis in the skin (Knaś et al. 2013, Kuźmiński et al. 2012, Żebrowska et al. 2005).

Matrix metalloproteinases comprise a family of over 20 structurally related proteins which are zincdependent and calcium-activated endopeptidases. The members of MMPs are able to degrade most extracellular matrix proteins and are involved in tissue remodeling and contribute to cell migration by eliminating extracellular matrix and basement membrane barriers. MMPs and their inhibitors play important roles in physiological processes such as embryogenesis and wound healing; however, these enzymes are also involved in the pathogeneses of many diseases, such as cancer, atherosclerosis or allergic diseases (Kuźmiński et al. 2012, Naduk-Kik and Hrabec 2008, Żebrowska et al. 2005). Metabolism of collagen in connective tissue remodeling process under various pathological conditions requires the interplay of MMPs, not only soluble ones (MMP-2, MMP-9) but also the membrane bound type 1 MMP (MT1-MMP) and TIMP-1, TIMP-2. The resulting complex MT1-MMP/ TIMP-2/MMP-2 initiates and completes the degradation of collagen fibril (Collier et al. 2011). Recent reports show that MMP-2 and MMP-9 may be involved in the pathogenesis of diabetes mellitus and diabetic complications such as diabetic retinopathy. MMP-9 has the ability to degrade type IV collagen, and insulin is able to activate IL-8, the main chemoattractant factor for neutrophils and monocytes. In addition MMP-9 enables inflammatory cell migration and pancreas colonization by eliminating the basement membrane barriers. MMP-18 (type IV collagenases) are important for endothelial cell migration occurring during neovascularization (diabetic retinopathy), as angiogenesis needs extracellular matrix degradation; furthermore, these enzymes are able to degrade the pigment epithelium-derived factor, which is 
the principal antiangiogenic protein of the eye (Naduk-Kik and Hrabec 2008). The MMP-3 (stromelysin 1, progelatinase) degrades collagen types: II, III, IV, IX, and X, proteoglycans, fibronectin, laminin, and elastin. In addition, MMP-3 can also activate other MMPs such as: MMP-1, MMP-7, and MMP-9; rendering MMP-3, crucial in connective tissue remodeling. The MMP-3 is thought to be involved in wound repair, progression of atherosclerosis, and tumor initiation (Ye et al. 1996). TIMP-2 is secreted by fibroblasts and endothelial cells and inhibits the activity of MMP-3, MMP-7 and MMP-9 (Trojanek 2012). MMP-3 can degrade telopeptides of interstitial collagens and denatured or hydrolyzed interstitial collagen (Fields 2013, Martins et al. 2013, Wu et al. 1991). Catabolism of interstitial collagen in extracellular matrix of several organs, including skin, is hydrolyzed not only by "classic" collagenases into $1 / 4$ and $3 / 4$ length fragments (MMP-1, MMP-8, and MMP-13), but also MMP-9 (gelatinase B) which cleaves type I and II collagen into $1 / 4$ and $3 / 4$ length fragments at the same cleavage site as classic collagenases (Fields 2013).

\section{Backround}

In the present literature, there is a lack of information on the metabolism of the extracellular matrix of the skin, in type 1 diabetes and insulin resistance. We wanted to assess the impact of type 1 diabetes and insulin resistance on the content of MMP-3, involved in the remodeling of the connective tissue of the dermis, characterized by content of type III collagen, as type I and III collagens are major components of the skin and plays an important role in the remodeling of the skin structure. The skin matrix metalloproteinase 3, tissue inhibitors of matrix metalloproteinase 2 and collagen III content changes in type 1 diabetes and insulin resistance treated with insulin and metformin were studied.

\section{Materials and Methods}

The study was approved by the Local Committee on the Ethical Use of Animals at the Medical University in Bialystok, Poland (N. 113/275/81 P). Adult male Wistar rats were obtained from the experimental animal house, Department of Experimental Pharmacology, Medical University in Bialystok. The rats weighed between $0.168-0.180 \mathrm{~kg}$ and were housed separately in standard conditions $\left(20-21^{\circ} \mathrm{C} \pm 2{ }^{\circ} \mathrm{C}, 12 \mathrm{~h}\right.$ of light/12 h of dark) with unlimited access to water (Knaś et al. 2013).

The rats were divided randomly into five groups of 8 rats each. Control rats (C) were injected intraperitoneally (i.p.) with $0.9 \% \mathrm{NaCl}$ (Knaś et al. 2013); the mean glycemia at the end of the experiment: $5.09 \pm 0.40 \mathrm{mmol} / 1$.

Type IDDM was induced in two groups of rats, by a single injection of STZ - Streptozocin (Sigma, USA; $5 \times 10^{-5} \mathrm{~kg} / \mathrm{kg}$, i.p. prepared in the citrate buffer $0.1 \mathrm{M}, \mathrm{pH} 4.5$ ): untreated diabetes type 1 (IDDM) and in insulin treated type 1 diabetes group (IDDM+I) (Al-Bayaty and Abdulla 2012). Blood was drawn from the tail on the seventh day after the STZ injection. The blood glucose level was used to confirm the development of diabetes: $20.21 \pm 0.86 \mathrm{mmol} / 1$. Treatment of diabetes was started in one group (IDDM+I) during the fifth week after the STZ injection. The rats were treated with insulin (6-8 U subcutaneously, depending on blood glucose level) once a day for 3 weeks (Knaś et al. 2013); the mean glycemia at the end of the experiment: $5.42 \pm 0.29 \mathrm{mmol} / 1$.

Insulin resistance was induced in two groups by a high-fat diet (HFD) (Hou et al. 2012, Knaś et al. 2013 ) with $60 \%$ fat, $20 \%$ protein, $20 \%$ carbohydrate: untreated insulin resistance (IR), metformin (M) treated insulin resistance $(\mathrm{IR}+\mathrm{M})$. The blood glucose level was used to confirm the development of diabetes: 19.20 $\pm 94.82 \mathrm{mmol} / \mathrm{l}$. Treatment of insulin resistance was started in one group (IR $+\mathrm{M}$ ) during the fifth week after the high-fat diabetes-inducing diet. The rats were treated with metformin $\left(3 \times 10^{-4} \mathrm{~kg}-5 \times 10^{-4} \mathrm{~kg} / \mathrm{kg}\right.$ per os $)$, depending on blood glucose levels once a day for 3 weeks (Knaś et al. 2013); the mean glycemia at the end of the experiment: $5.46 \pm 0.23 \mathrm{mmol} / \mathrm{l}$.

The tail blood was drawn on the seventh day after the HFD and the blood glucose levels were estimated using a glucometer (Accu-Check Active, Roche, France) (Knaś et al. 2013).

The animals were anesthetized by the injection of pentobarbital in a dose of $80 \mathrm{mg} / \mathrm{kg}$ of body weight. All the animals were put to sleep and sacrificed. Skin samples were taken from a shaved dorsal neck of each rat (approximately $2 \mathrm{~cm}^{2}$ ). Skin samples were rinsed in icecold PBS (0.01 mol/1, pH 7.0-7.2). Small pieces of tissue were homogenized 6 times in 5-10 $\mathrm{ml}$ of PBS with glasshomogenizer (Omni TH, Omni International, USA) on ice. The resulting suspension was sonicated with an ultrasonic cell disrupter (UP 400S, Hielscher, Germany); $1800 \mathrm{~J}$ per sample, $20 \mathrm{~s} \times 3$ on ice to further break the 
tissue fragment. After that, the homogenates were centrifuged for $5 \mathrm{~min}$ at $5000 \mathrm{x} \mathrm{g}$ (MPW 351, Poland) to remove the supernatant and assayed immediately (Knaś et al. 2013).

\section{Assays}

ELISA Kits (USCN Life Science, China) were used to measure content of matrix metalloproteinase 3 (ELISA Kit for Matrix Metalloproteinase 3 - MMP3), tissue inhibitor of matrix metalloproteinase 2 (ELISA Kit for Tissue Inhibitors of Metalloproteinase 2 - TIMP2) and content of collagen type 3 (ELISA Kit for Collagen Type III - COL 3). All performed in duplicate.

\section{Statistics}

The results were reported as median. Statistical analysis was performed using Statistica 10.0 (Statsoft, Cracow, Poland). The Kruskal-Wallis ANOVA test was used to study the significant differences between groups. The statistical significance was defined as $\mathrm{p}<0.05$.

\section{Results}

Content of matrix metalloproteinases 3 (MMP-3)

The MMP-3 content in the skin of untreated IDDM and IR rats was significantly higher in comparison to the MMP-3 content of the control skin $(C)(p<0.05)$. The MMP-3 content in diabetic rats treated with insulin $(\mathrm{IDDM}+\mathrm{I})$ and metformin treated insulin resistance rats $(\mathrm{IR}+\mathrm{M})$ was significantly higher as compared to the content in the skin of diabetic rats (IDDM) $(\mathrm{p}<0.001)$ and insulin resistance rats $(\mathrm{IR})(\mathrm{p}<0.05)$ (Table 1$)$.
Content of tissue inhibitors of matrix metalloproteinases 2 (TIMP-2)

The TIMP-2 content was significantly lower in the skin of diabetic rats (IDDM) $(\mathrm{p}<0.05)$ and rats (IR) $(p<0.05)$ in comparison to healthy rats $(C)$. The TIMP-2 content in the skin of diabetic rats treated with insulin (IDDM+I) and rats with metformin treated insulin resistance $(\mathrm{IR}+\mathrm{M})$ were significantly higher in comparison to the content in the skin of diabetic rats (IDDM) $(\mathrm{p}<0.05)$ and in the skin of rats with insulin resistance (IR) $(\mathrm{p}<0.05)$ (Table 1$)$.

\section{Ratio of MMP-3/TIMP-2}

The MMP-3/TIMP-2 ratio was significantly higher in the skin of diabetic rats (IDDM) $(\mathrm{p}<0.05)$ and insulin treated diabetic rats (IR) $(p<0.05)$ in comparison to healthy rats $(\mathrm{C})$. In diabetic rats treated with insulin $(\mathrm{IDDM}+\mathrm{I})$ and insulin resistant rats treated with metformin (IR+M) MMP-3/TIMP-2 the skin ratio was significantly lower compared to the MMP-3/TIMP-2 ratio in the skin of diabetic rats (IDDM) $(\mathrm{p}<0.001)$ and insulin resistant rats $(\mathrm{IR})(\mathrm{p}<0.05)$ (Table 1$)$.

\section{Content of collagen III (COL III)}

The type III collagen content was significantly lower in the skin of diabetic rats (IDDM) $(\mathrm{p}<0.001)$ and rats with insulin resistance (IR) $(p<0.001)$ in comparison to healthy rats $(\mathrm{C})$. The type III collagen content in the skin of diabetic rats treated with insulin (IDDM+I) and insulin resistant rats treated with metformin $(\mathrm{IR}+\mathrm{M})$ was significantly higher compared to the collagen III content in the skin of diabetic rats (IDDM) $(\mathrm{p}<0.001)$ and of insulin resistant rats (IR) $(\mathrm{p}<0.001)$ (Table 1$)$.

Table 1. The content of MMP-3, TIPM-2, MMP-3/TIMP-2, COL III in the rats' skin $\left(1.0 \times 10^{-6} \mathrm{~kg} / 10^{-4} \mathrm{~kg}\right.$ total of skin proteins).

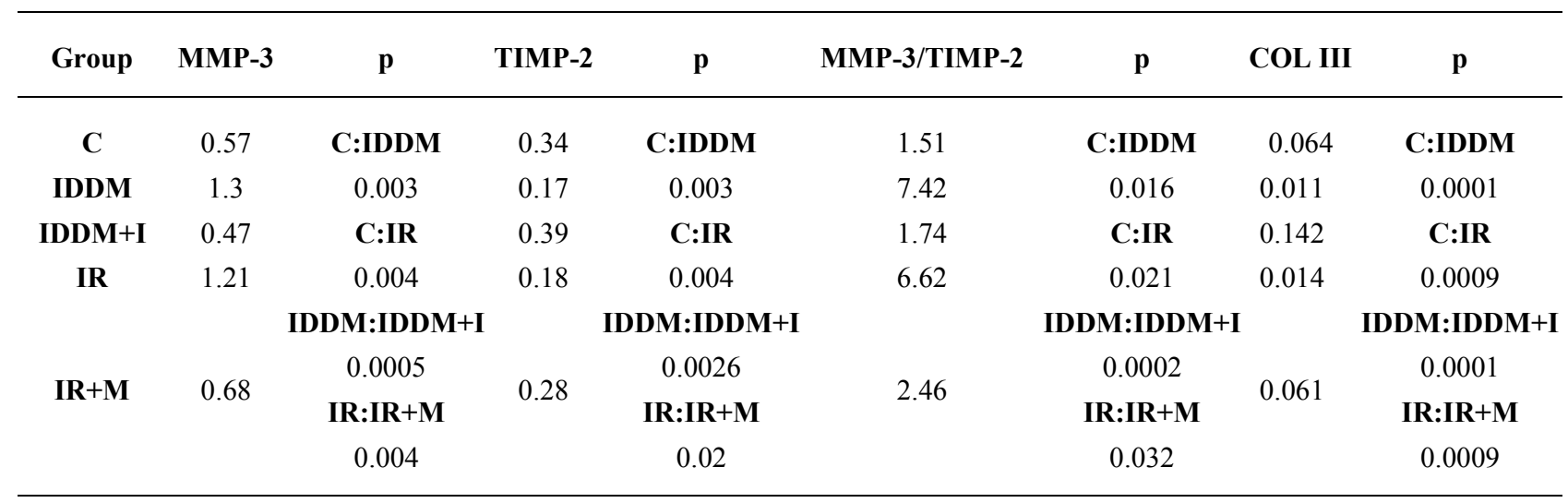

C - control group, IDDM - diabetes mellitus type 1 group, IDDM+I - diabetes mellitus type 1 treated with insulin group, IR - insulin resistance group, IR+M - insulin resistance treated with metformin group. Statistical significance defined as $p<0.05$. 


\section{Discussion}

Type I and III collagens are major components of the skin that play an important role in the remodeling of the skin structure. We selected the matrix metalloproteinase 3, due to participation of MMP-3 in catabolism of type III collagen (Jinnin 2010). Also, the interrelationships between the content of MMP-3 and TIMP-2 in the experimental model are not described in present literature.

Our results indicate that some parameters of homeostasis of the skin's extracellular matrix in diabetes and insulin resistance rats are different, comparing to the control rats. We have shown increased MMP-3 content and decreased TIMP-2 content in the skin of rats with diabetes and insulin resistance compared to the control. The significant MMP-3/TIMP-2 ratio increase and significant decrease of collagen type III content were observed. A relationship between the increase in the skin of MMP-3 content and decrease in content of collagen III indicate on extracellular matrix increased catabolism in the skin (Dalton 2005, Knaś et al. 2013). The increased ratio of MMP-3/TIMP-2 in the diabetic skin suggests an imbalance between the degradation and the synthesis of the extracellular matrix, evidenced by the significantly decrease in collagen III content. Our previous study on MMP-2, TIMP-3 and COL I (Knaś et al. 2013) has shown that excessive glycation of collagen damages the skin structure which was documented by the histological examination: a decrease of a skin thickness, disappearance of the multilayer epithelial structure of the epidermis, atrophy and degeneration of collagen fibers in the dermis, in comparison to the control rats. The similar changes were observed in our studies and also other studies (Chen 2009, Knaś et al. 2013).

Diabetic rats treated with insulin $($ IDDM $+\mathrm{I})$ and insulin resistance rats treated with metformin (IR $+\mathrm{M})$ resulted in a significant reduction of MMP-3 content in the skin and increased TIMP-2 and collagen content as compared to the untreated diabetic (IDDM) and with insulin resistant (IR) rats.

The content of MMP-3 in the skin of insulin treated diabetes type 1 (IDDM+I) and metformin treated insulin resistant rats (IR $+\mathrm{M})$ did not differ significantly from the control group. The same was observed according to the TIMP-2 content. This may be due to increased susceptibility to glycation and degradation of TIMP-2 (Knaś et al. 2013). Insulin and metformin treatment increased content of COL III compared to healthy rats, which may be due to the hypoglycemic and anabolic activity of insulin. It has been shown that insulin increases the uptake of amino acids in the cells, enhances protein synthesis; stimulates the RNA synthesis and polypeptides formation during translation (Miers and Barrett 1998, Sunahara et al. 2012). The increase of the collagen III content in the skin of insulin resistant rats treated with metformin (IR $+\mathrm{M})$, comparing to control level (C), may be the result of inhibiting the formation of advanced glycation products, due to normoglycemia. It was reported that normoglycemia restores the physiological metabolism of these proteins (Boyle et al. 2010, Caso and McNurlan 2010, Elgebaly et al. 2010, Lobman et al. 2002, Schneir et al. 1982, Spanheimer 1992, Verhofstad et al. 1998). Andreea et al. (2008) and Collier et al. (2011) suggests that in the mimicked diabetic conditions, the human fibroblasts, incubated with increasing amount of AGE modified BSA, up-regulated the procollagen $\alpha 1$ (III) mRNA expression to the great extent in comparison to procollagen $\alpha 2(\mathrm{I})$ mRNA expression, whereas in high D-glucose levels there was a down regulation tendency of procollagen $\alpha 1$ (III) mRNA. Disturbance to the collagen metabolism may reduce the proper content of collagen but rather, it would be expected, that group of animals with type 1 diabetes (IDDM) will exhibit higher amount of collagen type III, due to the relative increase of AGEs. Due to above facts for future studies it is recommend to determine rat interstitial collagenase, MMP-13, as well. Collagen type III cleavages are the products not only of MMP-13 but also of MT1-MMP, MMP-2 and MMP-9 complexes, regulated by TIMP-1 and TIMP-2 (Collier 2011).

MMPs and TIMPs have a direct or indirect impact on the homeostasis in diabetic and insulin resistance skin (excessive glycation of collagen damages the skin structure: a decrease of a skin thickness, disappearance of the multilayer epithelial structure of the epidermis, atrophy and degeneration of collagen fibers in the dermis) (Chen et al. 2009, Knaś et al. 2013). Increased activity of MMP-3 influences in collagen III content, which play an important role in tissue remodeling. MMP-3/TIMP-2 ratio in insulin treated diabetic rats skin (IDDM+I) and in insulin resistance with metformin treatment (IR $+\mathrm{M})$ are significantly lower in comparison to the skin of diabetic rats (IDDM) and rats with insulin resistance (IR), showing a decrease in protein catabolism in the skin of treated rats, that is in accordance with another reports (Elgebaly et al. 2010, Madibally et al. 2003, Schneir et al. 1982, Verhofstad et al. 1998). The above changes may underlie the prevalence of diabetic 
complications of the skin in the course of type 2 diabetes, in which insulin resistance is the first step in the development of the disease, and where the first choice oral drug is metformin (Muris et al. 2012, Perez and Kohn 1994, Van Hattem et al. 2008).

\section{Conclusions}

Type 1 diabetes and insulin resistance significantly reduced the quality of the skin, evidenced by the increase of MMP-3 content and a decrease of TIMP-2 content in the skin.

Type 1 diabetes and insulin resistance reduced the quality of the skin shown in a decrease of collagen III content in the skin, but it is recommended to determine interstitial collagenase MMP-13 content in the future studies.

Insulin and metformin treatment improved the quality of the diabetic skin reflected by the collagen III content increase.

\section{Conflict of Interest}

There is no conflict of interest.

\begin{abstract}
Abbreviations
C - control rats, COL III - type III collagen, ECM extracellular matrix, I - insulin, IDDM - type 1 diabetes mellitus, IR - insulin resistance, $\mathrm{M}$ - metformin, MMPs matrix metalloproteinases, MMP3 - matrix metalloproteinase 3, NIDDM - type 2 diabetes mellitus, TIMPs tissue inhibitors of matrix metalloproteinases, TIMP2 tissue inhibitors of matrix metalloproteinase 2 .
\end{abstract}

\section{References}

AL-BAYATY F, ABDULLA MA: A comparison of wound healing rate following treatment with aftamed and chlorine dioxide gels in streptozotocin-induced diabetic rats. Evid Based Complement Alternat Med 2012: 468764, 2012.

ANDREEA SI, MARIETA C, ANCA D: AGEs and glucose levels modulate type I and III procollagen mRNA synthesis in dermal fibroblasts cells culture. Exp Diabetes Res 2008: 473603, 2008.

BALOCH GH, MEMON NM, DEVRAJANI BR, IQBAL P, THEBO NK: Cutaneous manifestations of type-II diabetes mellitus. JLUMHS 7: 67-70, 2012.

BOYLE JG, MCKAY GA, FISCHER M: Drugs for diabetes: part 1 metformin. Br J Cardiol 17: 231-234, 2010.

CASO G, MCNURLAN MA: Effect of insulin on whole body protein metabolism in children with type 1 diabetes. Curr Opin Clin Nutr Metab Care 13: 93-96, 2010.

CASQUEIRO JU, CASQUEIRO JA, ALVES C: Infections in patients with diabetes mellitus: a review of pathogenesis. Indian J Endocrinol Metab 16: S27-S36, 2012.

CHEN X, LIN W, LU S, SHI Y, ZOU J, LIU Z, LIAO W: Insulin prevents latent skin lesions by inhibiting the generation of advanced glycation end products in Streptozotocin-induced diabetes rats. Endocr Pathol 20: 163-169, 2009.

COLLIER IE, LEGANT W, MARMER B, LUBMAN O, SAFFARIAN S, WAKATSUKI T, ELSON E, GOLDBERG GI: Diffusion of MMPs on the surface of collagen fibrils: the mobile cell surface-collagen substratum interface. PLoS One 6: e24029, 2011.

DALTON SJ, MITCHELL CD, WHITING CV, TARLTON JF: Abnormal extracellular matrix metabolism in chronically ischemic skin: a mechanism for dermal failure in leg ulcers. J Invest Dermatol 125: 373-379, 2005.

DE ALMEIDA TAMEGA A, MILANEZI PINHEIRO ARANHA A, MASSAKI GUIOTOKU M, MIOT LDB, MIOT HA: Association between skin tags and insulin resistance (in Portuguese). An Bras Dermatol 85: 25-31, 2010.

ELGEBALY MM, PRAKASH R, LI W, OGBI S, JOHNSON MH, MEZZETTI EM, FAGAN SC, ERGUL A: Vascular protection in diabetic stroke: role of matrix metalloprotease-dependent vascular remodeling. $J$ Cereb Blood Flow Metab 30: 1928-1938, 2010.

FIELDS GB: Interstitial collagen catabolism. J Biol Chem 288: 8785-8793, 2013.

HOU L, LIAN K, YAO M, SHI Y, LU X, FANG L, HE T, JIANG L: Reduction of n-3 PUFFAs, specifically DHA and EPA, and enhancement of peroxisomal beta-oxidation in type 2 diabetic rat heart. Cardiovasc Diabetol 11: 126, 2012.

JELINEK JE: Cutaneous manifestations of diabetes mellitus. Int J Dermatol 9: 605-617, 1994. 
JINNIN M: Mechanisms of skin fibrosis in systemic sclerosis. J Dermatol 37: 11-25, 2010.

KNAŚ M, NICZYPORUK M, ZALEWSKA A, CAR H: The unwounded skin remodeling in animal models of diabetes types 1 and 2. Physiol Res 62: 519-526, 2013.

KUŹMIŃSKI A, PRZYBYSZEWSKI M, GRACZYK M, BARTUZI Z: The role of extracellular matrix metalloproteinases and their inhibitors in allergic diseases. Postep Derm Alergol 5: 384-389, 2012.

LAMBA S, KRISHTUL A, TAN M-H, LEBWOHL MG: Acanthosis nigricans in a plaque of scleroderma on the back of a diabetic patient: A case report. Int J Dermatol 1: 45-47, 2005.

LOBMAN R, AMBROSCH A, SCHULTZ G, WALDMANN K, SCHIWECK S, LEHNERTET H: Expression of matrix-metalloproteinases and their inhibitors in the wounds of diabetic and non-diabetic patients. Diabetologia 45: 1011-1016, 2002.

MACKIEWICZ-WYSOCKA M, ARASZKIEWICZ A, SCHLAFFKE J, KUCZYNSKI S, MICEK I, ZOZULINSKAZIOLKIEWICZ D: Lower melanin content in the skin of type 1 diabetic patients and the risk of microangiopathy. Exp Clin Endocrinol 122: 231-235, 2014.

MADIBALLY SV, SOLOMON V, MITCHELL RN, VAN DE WATER L, YARMUSH ML, TONER M: Influence of insulin therapy on burn wound healing in rats. $J$ Surg Res 109: 92-100, 2003.

MARTINS VL, CALEY M, O'TOOLE EA: Matrix metalloproteinases and epidermal wound repair. Cell Tissue Res 351: 255-268, 2013.

MASHARANI U, GERMAN MS: Pancreatic hormones and diabetes mellitus. In: Greenspan's Basic \& Clinical Endocrinology. GARDNER D, SHOBACK D (eds), New York, McGraw-Hill Medical, 2011, chapter: 17. http://www.accessmedicine.mhmedical.com/content.aspx?bookid=380\&sectionid=39744057.

MIERS WR, BARRETT EJ: The role of insulin and other hormones in the regulation of amino acid and protein metabolism in humans. J Basic Clin Physiol Pharmacol 9: 235-253, 1998.

MURIS DM, HOUBEN AJ, SCHRAM MT, STEHOUWER CD: Microvascular dysfunction is associated with a higher incidence of type 2 diabetes mellitus: a systematic review and meta-analysis. Arterioscler Thromb Vasc Biol 32: 3082-3094, 2012.

NADUK-KIK J, HRABEC E: The role of matrix metalloproteinases in the pathogenesis of diabetes mellitus and progression of diabetes retinopathy (in Polish). Postepy Hig Med Dosw 62: 442-450, 2008.

PEREZ MI, KOHN SR: Cutaneous manifestations of diabetes mellitus. $J$ Am Acad Dermatol 30: 519-531, 1994.

POWERS AC: Diabetes mellitus. In: Harrison's Principles of Internal Medicine. KASPER DL, BRAUNWALD E, FAUCI AS, HAUSER SL, LONGO DL, JAMESON JL (eds), McGraw-Hill, New York, 2005, pp 2152.

SCHNEIR M, RAMAMURTHY N, GOLUB L: Skin collagen metabolism in the streptozotocin-induced diabetic rat. Enhanced catabolism of collagen formed both before and during the diabetic state. Diabetes 31: 426-431, 1982.

SPANHEIMER RG: Correlation between decreased collagen production in diabetic animals and in cells exposed to diabetic serum response to insulin. Matrix 12: 101-107, 1992.

SUNAHARA KK, SANNOMIYA P, MARTINS JO: Briefs on insulin and innate immune response. Cell Physiol Biochem 29: 1-8, 2012.

TAYLOR R: Insulin resistance and type 2 diabetes. Diabetes 61: 778-779, 2012.

TROJANEK J: Matrix metalloproteinases and their tissue inhibitors (in Polish). Postepy Biochem 58: 353-362, 2012.

WU JJ, LARK MW, CHUN LE, EYRE DR: Sites of stromelysin cleavage in collagen types II, IX, X, and XI of cartilage. $J$ Biol Chem 266: 5625-5628, 1991.

VERHOFSTAD MHJ, BISSELING TM, HAANS EMH, HENDRIKS T: Collagen synthesis in rat skin and ileum fibroblast is affected differently by diabetes-related factors. Int J Exp Pathol 79: 321-328, 1998.

YE S, ERIKSSON P, HAMSTEN A, KURKINEN M, HUMPHRIES SE, HENNEY AM: Progression of coronary atherosclerosis is associated with a common genetic variant of the human stromelysin-1 promoter which results in reduced gene expression. $J$ Biol Chem 271: 13055-13060, 1996.

ŻEBROWSKA A, BOGDAŃSKA M, WASZCZYKOWSKA E: Matrix metalloproteinases and adamalisyns in pathomechanism of pemphigoid. Postep Derm Alergol 6: 283-287, 2005. 